\title{
ON RINGS OF ANALYTIC FUNCTIONS
}

\section{LIPMAN BERS}

Let $D$ be a domain in the complex plane (Riemann sphere) and $R(D)$ the totality of one-valued regular analytic functions defined in $D$. With the usual definitions of addition and multiplication $R(D)$ becomes a commutative ring (in fact, a domain of integrity). A oneto-one conformal transformation $\zeta=\phi(z)$ of $D$ onto a domain $\Delta$ induces an isomorphism $f \rightarrow f^{*}$ between $R(D)$ and $R(\Delta): f(z)=f^{*}[\phi(z)]$. An anti-conformal transformation

$$
\zeta=\overline{\phi(z)}
$$

also induces an isomorphism:

$$
\overline{f(z)}=f^{*}[\overline{\phi(z)}] .
$$

The purpose of this note is to prove the converses of these statements.

THEOREM I. If $R(D)$ is isomorphic to $R(\Delta)$, then there exists either a conformal or an anti-conformal transformation which maps $D$ onto $\Delta .{ }^{1}$

THEOREM II. If $D$ and $\Delta$ possess boundary points, then every isomorphism between $R(D)$ and $R(\Delta)$ is induced by a conformal or an anticonformal transformation of $D$ onto $\Delta$.

Theorem I may be regarded as a complex variable analogue of theorems characterizing a topological space in terms of the family of its continuous functions. If $R(D)$ is made into a topological ring by defining $f_{n} \rightarrow f$ to mean that $f_{n}(z) \rightarrow f(z)$ uniformly in every bounded closed subset of $D$, then Theorem II implies that, except for a trivial special case, every isomorphism between $R(D)$ and $R(\Delta)$ is of necessity a homeomorphism.

To prove the theorems we consider a fixed isomorphism between $R(D)$ and $R(\Delta)$. It takes a function $f(z), z \in D$, into a function $f^{*}(\zeta)$, $\zeta \in \Delta$, a set $S \subset R(D)$ into a set $S^{*} \subset R(\Delta)$. Let $c$ be a complex constant.

Presented to the Society, November 2, 1946; received by the editors May 27, 1947.

1 After this paper was completed the author learned about a closely related unpublished result which was obtained by C. Chevalley and S. Kakutani several years ago. Chevalley and Kakutani proved that if to each boundary point $W$ of $B$ there exists a bounded analytic function defined in $B$ and possessing at $W$ a singularity then $B$ is determined (modulo a conformal transformation) by the ring of all bounded analytic functions. The author is indebted to Professor Chevalley for the opportunity of reading a draft of the paper containing the proof. 
For the sake of brevity we denote the element of $R(D)$ corresponding to the functions $f(z) \equiv c$ (or the element of $R(\Delta)$ corresponding to the function $g(\zeta) \equiv c$ ) by the letter $c$. We call a complex number rational if its real and imaginary parts are rational.

Lemma 1. Either $i^{*}=i$ and for every rational complex constant $r: r^{*}=r$, or $i^{*}=-i$ and $r^{*}=\bar{r}$.

The proof is clear.

Lemma 2. If $c$ is a constant, so is $c^{*}$.

PROOF. If $c$ is rational the assertion is contained in the preceding lemma. Irrational constants $c$ are characterized by the existence of the inverse of the element $c-r$ for every rational constant $r$.

LEMMA 3. All elements of $R(D)$ are constants if and only if $D$ is the whole complex plane including the point at infinity.

The proof is clear.

Lemmas 2 and 3 contain the proof of Theorem I for the case when $D$ is the domain $0 \leqq|z| \leqq \infty$. In what follows we consider only domains possessing boundary points. Without loss of generality we assume that neither $D$ nor $\Delta$ contains the point at infinity. We also assume that $i^{*}=i$; the case $i^{*}=-i$ can be treated in the same way.

We denote the set of all functions belonging to $R(D)$ and vanishing at a point $a \in D$ by $I_{a}$. The set $I_{\alpha} \subset R(\Delta), \alpha \in \Delta$, is defined similarly.

LEMma 4. There exists a one-to-one mapping $z \rightarrow z^{\prime}=\phi(z)$ of $D$ onto $\Delta$ such that $I_{a}^{*}=I_{\phi(a)}$.

Proof. Every element of $R(D)$ generates a principal ideal $(f)$, that is, the set of all elements of the form $f h, h \in R(D)$. $(f)$ is said to be a maximal principal ideal if $(f) \neq R(D)$ and if $(f) \subset(g) \neq R(D)$ implies that $(f)=(g)$. It is clear that $(f)^{*}=\left(f^{*}\right)$ and that $\left(f^{*}\right)$ is a m. p. ideal if and only if $(f)$ is. Hence Lemma 4 is an immediate consequence of the following lemma.

LeMma 5. $(f)$ is a m.p. ideal if and only if $(f)=I_{a}$.

Proof. $I_{a}$ is the principal ideal generated by the function $z-a$. If $I_{a} \subset(g) \neq R(D)$, then $g(z)$ must possess zeros in $D$. Since $z-a=g(z) h(z), h \in R(D), g(a)=0$ and $g \in I_{a}$. On the other hand, if $f(z)$ has no zeros in $D$, then $(f)=R(D)$, and if $f(a)=f^{\prime}(a)=0$, or if $f(a)=f(b)=0, a \neq b$, then $(f)$ is contained in and different from the 
principal ideal generated by the function $z-a$. It follows that $(f)$ is a m.p. ideal if and only if $f(z)=(z-a) e^{h(z)}, a \in D, h \in R(D)$.

LEMMA 6. For every point $z_{0} \in D, f\left(z_{0}\right)^{*}=f^{*}\left[\phi\left(z_{0}\right)\right]$.

Proof. If $c$ is a constant such that $f\left(z_{0}\right)=c$, then $c-f$ belongs to $I_{z_{0}}$, so that $c^{*}-f^{*}$ belongs to $I_{\phi\left(z_{0}\right)}$ and $f^{*}\left[\phi\left(z_{0}\right)\right]=c^{*}$.

The following two lemmas are immediate consequences of Lemma 6.

LEMMA 7. If $z_{0} \in D$ and $f\left(z_{0}\right)$ is a rational number, then $f\left(z_{0}\right)$ $=f^{*}\left[\phi\left(z_{0}\right)\right]$.

LEMMA 8. If $f(z)$ is univalent in $D$, then $f^{*}(\zeta)$ is univalent in $\Delta$.

LEMMA 9. Let $f(z)$ be a univalent function defined in $D$, let $f(D)$ be the image of $D$ under the transformation $w=f(z)$, and let $W$ be the (finite) limit of a convergent sequence of distinct rational points $\left\{w_{n}\right\}$ belonging to $f(D) . W$ is a boundary point of $f(D)$ if and only if there exists a function $g(z) \in R(D)$ such that $g\left[h\left(w_{n}\right)\right]=n, h(w)$ being the function inverse to $w=f(z)$.

Proof. If $W$ is a boundary point of $f(D)$, choose an entire function $F(Z)$ such that $F\left[\left(W-w_{n}\right)^{-1}\right]=n$. The function $g(z)$ $=F\left\{[W-f(z)]^{-1}\right\}$ satisfies the conditions of the lemma. On the other hand, if $W$ is an interior point of $f(D), W=f(a), a \in D$, and for every $g(z) \in R(D), \lim g\left[h\left(w_{n}\right)\right]$ exists and is finite.

LEMMA 10. Let $f(z)$ be a univalent function defined in $D$, so that $f^{*}(\zeta)$ is a univalent function defined in $\Delta$. The domains $f(D)$ and $f^{*}(\Delta)$ are identical.

Proof. It follows from Lemma 7 that the rational points belonging to $f(D)$ are identical with the rational points belonging to $f^{*}(\Delta) . f(D)$ is the set of all limit points of its rational points, except those limit points which lie on the boundary of $f(D)$. A similar remark applies to $f^{*}(\Delta)$. But Lemmas 7 and 9 imply that if a sequence of rational points from $f(D)$ converges to a boundary point $W, W$ is a boundary point of $f^{*}(\Delta)$.

Lemma 10 contains the proof of Theorem I for domains possessing boundary points.

The proof of Theorem II depends on the following lemma.

LEMMA 11. If $D$ possesses boundary points, then $c^{*}=c$ for every constant $c$.

We prove this lemma in several steps. Let $B$ be any domain. By 
$m[B]$ we denote the set of all complex numbers $d$ such that the translation $Z=z+d$ maps $B$ onto itself.

LEMMA 12. For every univalent function $f \in R(D)$ and for every constant $c$ the difference $c-c^{*}$ belongs to $m[f(D)]$.

Proof. The function $f_{1}=f+c$ is univalent in $D$, and the functions $f^{*}$ and $f_{1}^{*}=f^{*}+c^{*}$ are univalent in $\Delta$ (Lemma 8). By virtue of Lemma $10, f(D)$ is identical with $f^{*}(\Delta)$, and $f_{1}(D)$ is identical with $f_{1}^{*}(\Delta)$. But a translation by $c$ takes $f(D)$ into $f_{1}(D)$ and a translation by $-c^{*}$ takes $f_{1}^{*}(\Delta)$ into $f^{*}(\Delta)$. Thus the translation by $c-c^{*}$ leaves $f(D)$ invariant.

LEMMA 13. If $D$ possesses finite boundary points, then there exists a univalent function $f \in R(D)$ such that $m[f(D)]$ is a discrete set.

Proof. Let $f$ be a fixed univalent function defined in $D$. The set $m[f(D)]$ is closed and a modul, that is, it contains $d_{1}-d_{2}$ whenever it contains $d_{1}$ and $d_{2}$. Assume that $m[f(D)]$ is not discrete. Then it either contains all points, or all points of a straight line. If $W$ is a finite boundary point of $f(D)$, every point $W+d, d \in m[f(D)]$, is a boundary point. It follows that the boundary of $f(D)$ contains a finite straight segment $S$. Let $Z(w)$ be the function which maps the domain exterior to $S$ conformally onto $|Z|<1$. The function $g(z)=Z[f(z)]$ is univalent and bounded in $D$. It follows that $m[g(D)]$ contains only the point 0 .

Now we can prove Lemma 11 under the hypothesis of Lemma 13. Let $f \in R(D)$ be univalent and such that $m[f(D)]$ is discrete. For every positive number $t$ set $f_{t}=t f$. By Lemma 12 the difference $c-c^{*}$ belongs to $m\left[f_{t}(D)\right]$, that is, the number $\left(c-c^{*}\right) / t$ belongs to $m[f(D)]$. It follows that $c-c^{*}=0 .{ }^{2}$

It remains to establish Lemma 11 for the case of the whole finite plane.

Lemma 14. If $D$ is the domain $|z|<\infty$ and $\Delta$ the domain $|\zeta|<\infty$, then $c_{n} \rightarrow \infty$ implies $c_{n}^{*} \rightarrow \infty$.

Proof. Set $f(z)=z$. Then $f^{*}(\zeta)$ is univalent in $\Delta$, that is, $f^{*}(\zeta)$ $=A \zeta+B, A, B=$ const., $A \neq 0$. For this $f$ and for $z_{0}=c$, Lemma 6 yields $c^{*}=A \phi(c)+B$. Hence $c_{n}^{*} \rightarrow \infty$ whenever $\phi\left(c_{n}\right) \rightarrow \infty$. But $c_{n} \rightarrow \infty$

${ }^{2}$ An alternative argument was suggested to the author by $C$. Loewner. Assume that $c-c^{*}=r e^{i \theta}, r \neq 0$. It is easy to see that for any univalent $f$ and for any domain $D$ satisfying the hypothesis of Lemma 13 there exists a (finite or semi-infinite) straight segment $S$ whose interior points belong to $f(D)$ and whose end points are boundary points of $f(D)$. By a linear transformation we can achieve that $S$ be the semi-infinite segment $z=t e^{i \theta}, t>0$. It follows that $c-c^{*}$ does not belong to $m[f(D)]$. 
implies the existence of a function $g \in R(D)$ such that $g\left(c_{n}\right)$ is rational and $g\left(c_{n}\right) \rightarrow \infty$. By Lemma $7, g^{*}\left[\phi\left(c_{n}\right)\right] \rightarrow \infty$, from which it follows that $\phi\left(c_{n}\right) \rightarrow \infty$.

In order to prove Lemma 11 under the hypothesis of Lemma 14 we note that the transformation $c \rightarrow c^{*}$ is an automorphism of the complex field. This automorphism is continuous by virtue of Lemma 14 . Hence $c^{*}=c$, for we assumed that $i^{*}=i$.

Lemma 11 being established, Lemma 6 yields the following lemma.

LEMma 15. If $D$ possesses boundary points, then $f\left(z_{0}\right)=f^{*}\left[\phi\left(z_{0}\right)\right]$ for every $z_{0} \in D$.

This lemma would contain Theorem II if we would know that $\phi(z)$ is analytic in $D$. To show this we select for $f$ the function $f(z)=z$. Lemma 15 shows that $\phi$ is the function inverse to the univalent (analytic) function $f^{*}$.

In the statement of Theorem I the ring of all analytic functions cannot be replaced by the subring $B(D)$ of all bounded analytic functions defined in $D$, even if $B(D)$ is treated as a normed ring (with $\|f\|=1$.u.b. $|f(z)|)$, and the isomorphism between $B(D)$ and $B(\Delta)$ is required to be norm preserving. ${ }^{3}$ In fact, let $D$ be the domain $|z|<1$, and let $\Delta$ be the domain $0<|z|<1$. The normed rings $B(D)$ and $B(\Delta)$ are identical. ${ }^{4}$

Neither is it possible to replace $R(D)$ by the linear space $L(D)$ of all analytic functions defined in $D$, even if $L(D)$ is considered to be a topological space (with the topology defined above) and the isomorphism between $L(D)$ and $L(\Delta)$ is required to be a homeomorphism.

In fact, let $D$ be the domain $0<r<|z|<1$, and let $D_{1}$ and $D_{2}$ denote the domains $|z|<1$ and $|z|>r$, respectively. Every function $f \in L(D)$ admits a unique Laurent decomposition: $f(z)=g(z)+h(z) / z$, $g \in L\left(D_{1}\right), h \in L\left(D_{2}\right)$. It is easy to see that $f_{n} \rightarrow f$ if and only if $g_{n} \rightarrow g$ and $h_{n} \rightarrow h$. On the other hand, the linear subspace of $L\left(D_{2}\right)$ consisting of all functions of the form $h(z) / z, h \in L\left(D_{2}\right)$, is topologically isomorphic to $L\left(D_{2}\right)$, and $L\left(D_{2}\right)$ is topologically isomorphic to $L\left(D_{1}\right)$. It follows that $L(D)$ is topologically isomorphic to the direct sum of two spaces $L\left(D_{1}\right)$. Thus $L(D)$ considered as an abstract linear topological space is independent of $r$.

\section{SYRACUSE UNIVERSITY}

\footnotetext{
${ }^{3}$ As a matter of fact, every isomorphism is.
}

${ }^{4}$ Cf., however, footnote 1 . 